\title{
Long-term results of functional endoscopic sinus surgery in children with chronic rhinosinusitis with nasal polyps*
}

\author{
Marjolein E. Cornet, Christos Georgalas, Susanne M. Reinartz, \\ Wytske J. Fokkens
}

Department of Otorhinolaryngology, Academic Medical Centre Amsterdam, Amsterdam, The Netherlands
Rhinology 51: 328-334, 2013

DOl:10.4193/Rhino13.079

*Received for publication:

May 30, 2013

Accepted: July 14, 2013

\section{Summary}

Background: Chronic rhinosinusitis with nasal polyps (CRSwNP) is rare in children and has a major impact on Quality of Life (QoL). Functional endoscopic sinus surgery (FESS) has proven to be an effective treatment, but it is still unclear what long-term outcomes are in children with CRSwNP. The objective of this study was to assess long-term results of FESS in children with CRSwNP.

Methodology: We performed a combined prospective and retrospective study. A QoL questionnaire was send to all children with CRSwNP who received FESS between the year 2000-2010. Almost half of these children also filled in this questionnaire preoperatively.

Results: Forty-four Children underwent FESS. From 18 patients, we also prospectively collected preoperative QoL questionnaires. The mean follow-up period was 4.0 years $( \pm 2.9)$. The mean age at surgery was 13 years $( \pm 2.9)$. Of these children, 9 had CF and 10 children asthma. R-SOM scores showed a significant improvement both in general symptoms as well as several different domains when comparing pre- and postoperative questionnaires. Only 5 of 44 patients needed a subsequent intervention. In children with CF this was 3 of 9.

Conclusion: This study demonstrates that long-term results of FESS in children with CRSWNP are good. QoL has improved significantly, especially in nasal symptoms, showing that FESS is a good treatment in children with CRSwNP. Furthermore, even children with CF show good results.

Key words: endoscopic sinus surgery, children, nose, paranasal sinuses, quality of life, nasal polyps

\section{Introduction}

CRS with nasal polyps (CRSwNP) is rare in children and has a major impact on the Quality of Life (QoL) of paediatric patients and their parents ${ }^{(1)}$. Because of the physical and psychological consequences of CRSwNP in children, a thorough treatment is needed.

In adults with CRSwNP functional endoscopic sinus surgery (FESS) is considered to be the treatment of choice when maximum medical treatment fails ${ }^{(2,3)}$. Several studies have shown that most patients benefit from this approach and that there is a revision rate of $20 \%{ }^{(4,5)}$. In children with CRSwNP on the other hand, surgical success rates are not known.

Until now there are some studies published describing the results of FESS in children, but they mainly focus on the results in children with CRS without nasal polyps (CRSsNP) and they report contradictory outcomes ${ }^{(6-13)}$. A meta-analysis performed by Hebert and Bent showed positive outcome in $88.7 \%$ of 832 children with CRSsNP who underwent FESS with an average follow up of 3.7 years ${ }^{(8)}$. Also several studies indicate that there is significant improvement in QoL after FESS in children with CRSSNP ${ }^{(6,11)}$. Besides, overall safety of FESS in children with CRSsNP has been established in some case series ${ }^{(14,15)}$. 
Up to now, all studies on results of paediatric FESS included mainly children with CRSsNP. Only a small number of children that were included had CRSWNP. The largest study describes the results of FESS in 51 children with CRSwNP ${ }^{(16)}$. The main objective was to determine appropriate duration of postoperative evaluation and they concluded that it should be performed for 4 years up to an age of at least 12 years. Interestingly, they found that CT images at 1 year after surgery were rated as unchanged or worsened in approximately half of the patients. However, at 4 years after operation nearly all patients were rated as improved or better on CT images. A long-term, retrospective study was performed by Siedek et al. who focused on the prognostic factors in FESS ${ }^{(7)}$. In total, 115 children were included and response rate was $64 \%(73 / 115)$. Of these 73 children with a mean age of 12 years, 39.1\% had CRSwNP, 51.3\% had CRSsNP, 5.2\% had a maxillary cyst and $4.3 \%$ an antrochoanal polyp. They reported a $76 \%$ improvement at 5.4 years after FESS and several negative prognostic factors were found, including: cystic fibrosis, asthma, nasal polyps, allergies, previous surgery and smoking.

Furthermore it has been reported that the majority of children with CRSwNP has cystic fibrosis (CF) ${ }^{(17)}$.

Even though several studies addressed positive outcomes after FESS in children with CF, usually length of follow-up is limited.

(18-21) CF is still a chronic disease of mucociliary transport and even after successful surgery problems like infections or nasal polyps can recur. Because exact results of FESS in children with CF are not known, it can lead to a more negative attitude of surgeons to perform FESS in children with CF.

The main objective of this study was to assess long-term results of FESS in children with CRSwNP with and without CF and to determine outcome, symptoms, quality of life and complications.

\section{Materials and methods}

\section{Patients}

We included 44 children with bilateral CRSwNP (aged 6-18 years) undergoing FESS between the year 2000 and 2010 in the Department of Otorhinolaryngology of the Academic Medical Centre in Amsterdam, the Netherlands. Approval to sent questionnaires to the participants was obtained from the local ethical committee. Children with antrochoanal polyps or inverted papillomas were excluded from the study.

\section{Study design}

A QoL questionnaire was send to all (44) children who received FESS because of CRSwNP. Herefore we used the Rhinosinusitis Outcome Measure (RSOM-31). Almost half (18) of these children also filled in this questionnaire preoperatively. We first performed a prospective analysis in which we compared the preoperative results to the postoperative results to look at possible improvement after FESS in children.

Secondly, we performed a retrospective analysis of the QoL at long-term follow-up after FESS in all children. We calculated the mean post-operative RSOM score for the whole group and when analyzing these findings, we compared results of children with CF or asthma to children without CF or asthma.

Furthermore, we distinguished 4 subgroups according to mean RSOM score points to make the analysis easier. Group I (0-1 points) consisted of patients with no or little symptoms, group II (2 points) little to moderate symptoms, group III (3 points) moderate to severe symptoms and group IV (4 points) severe to extreme symptoms. Besides this, we separately analysed the specific nasal domain of the R-SOM score, consisting of the first 6 questions.

Moreover, we performed a retrospective analysis of medical records of the children, including: age, sex, medical history, allergies, asthma, CF (sweattest and/or genetic tests), smoking, presenting symptoms, use of medication (steroids), CT scan, type of surgery, prior operations, complications and need for revision surgery.

\section{Surgery performed}

FESS was performed in all children after failure of optimal medical treatment consisting minimally of topical corticosteroids and saline irrigation for at least three months and sometimes also including oral antibiotics and systemic corticosteroids. All children had a preoperative CT scan. The extent of the surgery was tailored to the extent of the disease and always consisted of infundibulotomy and partial ethmoidectomy and sometimes included more extended ethmoidectomy and/or sphenoidectomy. In most cases a microdebrider was used during surgery ${ }^{(22)}$. Frontal sinus surgery was not performed in this patient group. All operative details and complications were recorded. Postoperatively patients received topical corticosteroid treatment and saline irrigation. Furthermore, we recorded the need for revision surgery.

\section{Postal questionnaires}

Questionnaires were filled in preoperatively by part of the patients (18/44). Most of the time, the patients were old enough to fill out the questionnaires themselves, but sometimes there was assistance needed from the parents and/or the physician. The same questionnaires were sent out to all 44 patients postoperatively and consisted of several items. We measured quality of life (QoL) with the RSOM-31 questionnaire ${ }^{(23)}$. The RSOM-31 assesses 6 nasal symptoms (congestions, rhinorrhea, sneezing, hyposmia, postnasal discharge and thick nasal debris) and 25 other symptoms summarized in several domains: non-nasal, sleep-disorders, emotional symptoms, practical problems and general symptoms with a symptom score from 1-5. Furthermore, we asked every patient the question how nasal symptoms are now compared to before surgery on a 5 point scale ( $1=$ much better, $5=$ much worse). 


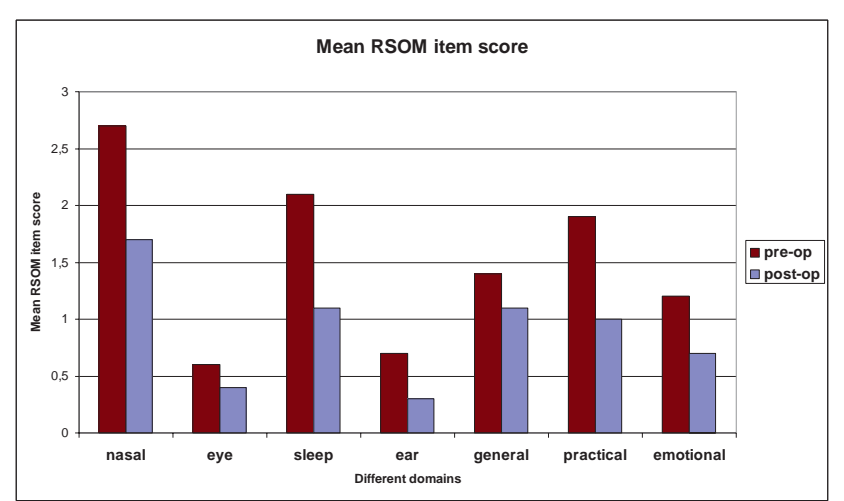

Figure 1. Changes in different domains of pre- and postoperative RSOM item scores $(n=18)$.

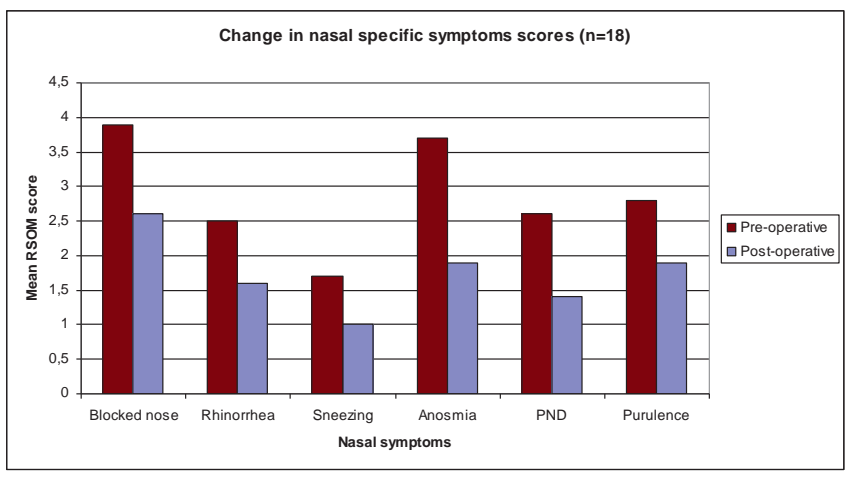

Figure 2. Change in nasal specific symptom scores (R-SOM) analysed in different groups $(n=18)$.

\section{Statistical analyses}

Statistical analysis was performed in SPSS and a p value $\leq 0.05$ was considered significant. We compared the pre- and postoperative scores using the Mann-Whitney $\mathrm{U}$ test. In the retrospective analysis, RSOM-31 scores were compared using the paired t-test if there was a normal distribution or otherwise the Mann-Whitney $\mathrm{U}$ test.

\section{Results}

\section{Statistical}

In total, 44 children underwent FESS due to CRSwNP. From 18 of these patients, we prospectively collected preoperative QoL questionnaires. Response rate of postoperative questionnaires was $82 \%$ ( 36 out of 44 ) and these 36 children ( 16 boys, 20 girls) constitute the final study group. Mean follow-up period of the whole group was 4.0 years (1-12 years). The follow-up period in children with CRWwNP without CF was 3.0 years (1-9 years) and in children with CF 6.0 years (3-12 years). Mean age at time of surgery for the whole group was 13 years old $( \pm 2.9)$.

Main preoperative symptoms were anosmia (100\%), nasal congestion (95\%) and rhinorrhea (83\%). About half of the children experienced headache (49\%) and cough (44\%).

Of these 36 children who underwent FESS because of CRSwNP, 9 children had CF (25\%) and 10 children asthma (28\%). Other predisposing factors were allergy (25\%), which was tested using a skin prick test, smoking (6\%) and aspirine intolerance (3\%).

\section{Outcome of FESS}

Eighty-six percent of the patients had a positive outcome after FESS. In total $14 \%$ (5) of the children needed a revision at time of follow up. Of the children with CF 3/9 (33\%) had a revision and of the children without CF only 2/27 (7\%). Revision surgery in children with CF was performed at 2, 3 and 7 years after primary surgery and in children without CF 2 and 4 years later.
Peri-operatively no complications occurred.

Postoperatively of all the patients $66.7 \%$ (24/36) still uses intranasal steroids at time of follow-up.

Prospective results $(\mathbf{n}=\mathbf{1 8})$

RSOM scores showed a significant improvement both in general symptoms as well as several different domains when comparing pre- and postoperative questionnaires. In total, $77 \%$ of the patients showed improvement in RSOM scores at long term follow-up.

Mean total pre-operative RSOM score was 43.4 and mean total postoperative score in was $31.3(p=0.04)$. There was no significant difference in outcome between male and female patients. If we look at CF patients in this group $(n=4)$, we could not find a difference in reduction of total RSOM score because the number of patients was too small.

Furthermore, when analyzing the total RSOM scores of the different domains, nasal symptoms $(p<0.01)$, sleep $(p=0.04)$ and practical functioning $(p=0.004)$ showed significant improvement. The total mean RSOM scores and domain scores are shown in Table 1. In Figure 1 RSOM scores $(n=18)$ are compared.

Furthermore, it was found that from the subset of nasal specific RSOM scores ( 6 parameters) 4 showed a significant improvement $(p<0.05)$ in the whole group: blocked nose, rhinorrhea, anosmia and post nasal drip (PND). Of children with CF, the same parameters improved (Figure 2).

\section{Retrospective results: whole group after FESS $(n=36)$}

Using a 5-point scale, a total of $78 \%$ of the patients reported overall improvement after FESS (score 1-2). In the CF group, 75\% of patients reported that their overall nasal symptoms are much better. There are only 2 patients with CF who reported the nasal symptoms being the same as before surgery and none of them 
Table 1. Mean pre- and postoperative RSOM scores compared ( $n=18)$.

\begin{tabular}{|c|c|c|c|c|c|}
\hline & $\begin{array}{l}\text { Preoperative score } \\
\text { mean ( } \pm \text { SD) }\end{array}$ & $\begin{array}{l}\text { Postoperative score } \\
\text { mean ( } \pm \text { SD) }\end{array}$ & $\begin{array}{r}95 \% \text { Con } \\
\text { Inter }\end{array}$ & $\begin{array}{l}\text { fidence } \\
\text { rval }\end{array}$ & $\begin{array}{c}\text { Sig. } \\
\text { (p-value) }\end{array}$ \\
\hline Total score & $43.4(18.4)$ & $31.3(21.8)$ & -23.6 & -0.5 & 0.04 \\
\hline Mean Item score & $1.4(0.6)$ & $1.0(0.7)$ & -0.7 & -0.1 & 0.04 \\
\hline Nasal item score & $2.8(0.6)$ & $1.7(1.2)$ & 0.7 & 1.5 & 0.0001 \\
\hline Eye item score & $0.6(1.0)$ & $0.4(0.8)$ & -0.5 & 0.7 & 0.73 \\
\hline Sleep item score & $2.1(1.8)$ & $1.1(1.1)$ & 0.01 & 1.9 & 0.04 \\
\hline Ear item score & $0.7(0.9)$ & $0.3(0.7)$ & -0.03 & 0.8 & 0.07 \\
\hline General symptoms & $1.4(1.0)$ & $1.1(0.9)$ & -0.3 & 0.8 & 0.30 \\
\hline Practical problems & $1.9(1.2)$ & $1.0(1.2)$ & 0.3 & 1.5 & 0.004 \\
\hline Emotional consequences & $1.2(1.3)$ & $0.7(1.1)$ & -0.3 & 1.3 & 0.21 \\
\hline
\end{tabular}

Table 2. Mean postoperative RSOM scores graded in four subgroups $(n=36)$.

\begin{tabular}{|c|c|c|c|c|c|}
\hline Group & $\begin{array}{c}\text { Mean } \\
\text { RSOM score }\end{array}$ & Level of symptoms & All children $(n=36)$ & $\underset{(n=9)}{C F}$ & $\begin{array}{c}N P \\
(n=27)\end{array}$ \\
\hline I & 0 and 1 & No or little & $24(67 \%)$ & $7(78 \%)$ & $17(63 \%)$ \\
\hline$\|$ & 2 & Little to moderate & $9(25 \%)$ & $1(11 \%)$ & $8(30 \%)$ \\
\hline III & 3 & Moderate to severe & $3(8 \%)$ & $1(11 \%)$ & $3(7 \%)$ \\
\hline IV & 4 and 5 & Severe to extreme & 0 & 0 & 0 \\
\hline
\end{tabular}

Table 3. Mean postoperative RSOM scores of the nasal domain.

\begin{tabular}{|c|c|c|c|c|c|}
\hline Group & $\begin{array}{l}\text { Mean nasal } \\
\text { RSOM score }\end{array}$ & Level of symptoms & All children $(n=36)$ & $\begin{array}{c}\text { CF } \\
(n=9)\end{array}$ & $\begin{array}{c}N P \\
(n=27)\end{array}$ \\
\hline I & 0 and 1 & No or little & $15(42 \%)$ & $5(56 \%)$ & $10(37 \%)$ \\
\hline II & 2 & Little to moderate & $11(30 \%)$ & $2(22 \%)$ & $9(33 \%)$ \\
\hline III & 3 & Moderate to severe & $9(25 \%)$ & $2(22 \%)$ & $7(26 \%)$ \\
\hline IV & 4 and 5 & Severe to extreme & $1(3 \%)$ & 0 & $1(4 \%)$ \\
\hline
\end{tabular}

reports the symptoms being worse (Figure 3).

The mean RSOM item score of the whole postoperative patient group ( $n=36$ ) was $1.16(S D \pm 0.87)$. The mean postoperative RSOM item score of the prospective group $(n=18)$ was 1.01 (SD $\pm 0.70)$. For the children with $C F(n=9)$ the mean post-operative
RSOM score was 0.91 (SD \pm 0.99$)$ and children without CF ( $\mathrm{n}=$ 27) 1.25 ( $S D \pm 0.83$ ). There was no significant difference between these postoperative outcomes $(p=0.35)$.

Furthermore when analyzing the difference in outcome of mean nasal item RSOM score between children with and without 


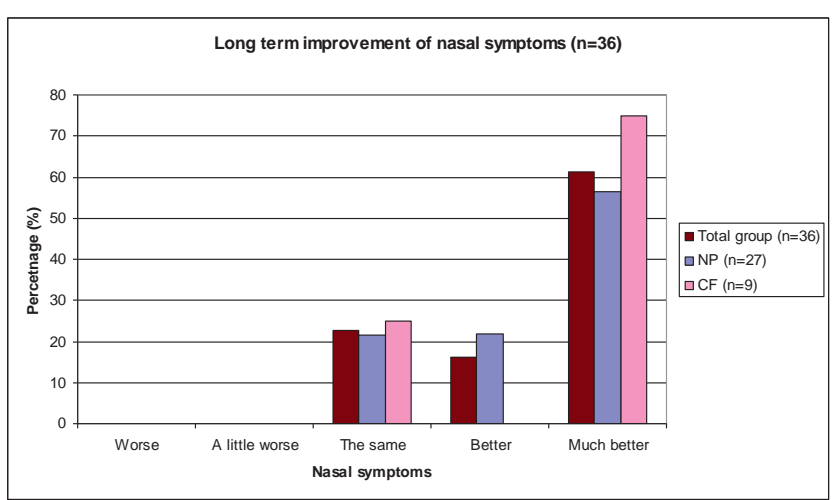

Figure 3. Long term improvement of nasal symptoms by self assessment after FESS $(n=36)$.

asthma, we find a significant better score in children without asthma (mean item score $=1.5$ ) than in children with asthma (mean item score $=2.3)(p=0.048)$.

If we look at the separate subgroups we created, $67 \%$ of the whole group belonged to subgroup I (no - little symptoms) and $25 \%$ to subgroup II (little - moderate symptoms). Only $8 \%$ had moderate to severe symptoms (subgroup III) and $0 \%$ was in subgroup IV (severe - extreme symptoms) (Table 2 and Figure 4A). The children with CF showed very good results compared to the whole group at follow-up. Out of the 8 children with CF, 7 (78\%) were postoperatively in the group with no or little nasal symptoms.

The results of the specific nasal postoperative RSOM scores are shown in Table 3 and Figure 4B.

\section{Discussion}

CRSwNP has a severe impact on QoL of adults and paediatric patients and can be difficult to treat ${ }^{(1)}$. The consensus is that surgical intervention should be considered in paediatric patients with CRSwNP when maximum medical therapy has failed ${ }^{(9)}$. Interestingly the data on paediatric FESS in patients with CRSwNP are very limited. This study shows that FESS is a very effective and safe treatment in children with CRSwNP.

Our study shows significant improvement in QoL at long-term follow-up after FESS in children with CRSwNP. In total, 78\% of the patients reported an overall improvement in RSOM score over time.

In our prospective analysis, RSOM scores showed significant improvement both in general symptoms as well as several different domains. Comparable results were found using the postoperative RSOM questionnaire showing that around $75 \%$ of the
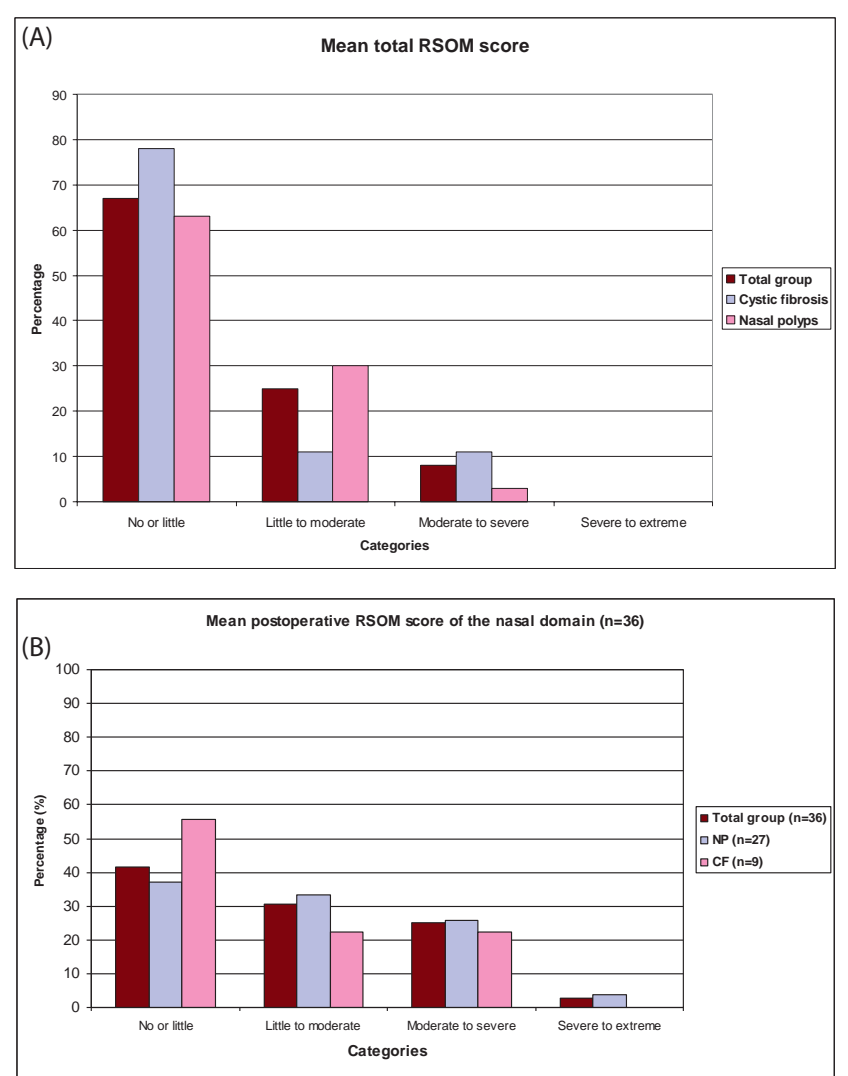

Figure 4. (A) Mean postoperative total R-SOM score $(n=36)$. (B) Mean postoperative R-SOM score of the nasal domain $(n=36)$.

children with CRSwNP were in the group with no or little or little to moderate symptoms at long-term follow-up. Moreover, from the subset of nasal specific RSOM scores, 4 out of 6 parameters showed significant improvement $(p<0.05)$ postoperatively. These results are comparable to the study from Siedek et al. ${ }^{(7)}$.

Furthermore, if we look at re-intervention rate, there was a revision surgery rate of $14 \%(5)$ in the whole group. We found that $\mathrm{CF}$ is a predictor for revision surgery. In the group of children with CF, 3 out of 9 CF patients ( $33 \%$ ) needed another operation after primary FESS and of the children without CF only 2 out of 27 (7\%). This means that CF might be a predictor for revision surgery and these children should be carefully monitored by an ENT specialist.

In contrary to common beliefs, in our group of children with CRSwNP who underwent FESS was only a small percentage of children with CF (25\%). We see that children with CF, although they have more recurrences, have good improvement of symptoms after FESS comparable to the rest of the group. Out of 9 children with CF, 6 (78\%) were postoperatively in the group with no or little nasal symptoms. Because our measurements were subjective using questionnaires, there could be a bias in 
answers from children with CF. It could be that the standard of health in a child with CF is generally different than in 'non' CFchildren. This could mean that nasal symptoms in children with CF can seem less important to them compared to their general health and therefore they might fill in a lower score on the RSOM questionnaire.

Asthma is commonly associated with CRSWNP in the paediatric population and therefore may influence FESS outcomes. Prevalence of asthma in children with CRSwNP is much higher than in the normal population. In our study the prevalence of asthma was $28 \%$ compared to around $10 \%$ in normal childhood population ${ }^{(24,25)}$.

When analyzing our results, we found a difference in outcome between children with and without asthma. Mean postoperative nasal RSOM score in asthma patients was significantly higher than in children without asthma $(p=0.048)$. However, there is lack of good randomized controlled studies describing the relationship between asthma in children and CRSwNP.

The strength of our study is the long follow-up period of approximately 4 years and the partly prospectively collected data. A limitation of this study is that not all data are prospectively collected. We only have preoperative questionnaires from half the patient group. Also were our outcomes of surgery measured by subjective questionnaires, not physical examination and therefore could be biased. Besides that, other factors that have not been assessed in this study, like socioeconomic factors and patient expectations, could have played a role. Nevertheless, the promising results in this study show benefits of FESS for the treatment of children with CRSwNP, even in children with CF.

\section{Concluding remarks}

We can conclude that long-term results of FESS in children with CRSwNP are good. Overall QoL has improved significantly for the whole group, especially in the domain of nasal symptoms, showing that FESS is also a very good treatment in children with CRSwNP. Furthermore, even children with CF show good results at long-term follow-up, besides the fact that $33 \%$ of the children with CF needed revision surgery in the long term. In literature until now was very little evidence at all about the efficacy of FESS specifically in children with CRSwNP, therefore we advice that more prospective studies should be performed to see if our results of FESS in children with CRSwNP are justified.

\section{Acknowledgements}

This study was not supported by any company or persons other than the authors.

\section{Authorship contribution}

MEC: designed the study, analyzed the data and wrote the manuscript; CG/SMR: critically read and commented on the paper; WJF: designed the study, analyzed the data and critically read and commented on the paper.

\section{Conflict of interest}

None declared.

\section{References}

1. Cunningham JM, Chiu EJ, Landgraf JM, Gliklich RE. The health impact of chronic recurrent rhinosinusitis in children. Arch Otolaryngol Head Neck Surg. 2000 ; 126: 1363-1368.

2. Alobid I, Benitez P, Valero A, Munoz R, Langdon C, Mullol J. Oral and intranasal steroid treatments improve nasal patency and paradoxically increase nasal nitric oxide in patients with severe nasal polyposis. Rhinology. 2012; 50: 171-177.

3. Fokkens WJ, Lund VJ, Mullol J, Bachert C, Alobid I, Baroody F, et al. European Position Paper on Rhinosinusitis and Nasal Polyps 2012. Rhinol Suppl. 2012; (23): 3-298.

4. Kennedy DW. Prognostic factors, outcomes and staging in ethmoid sinus surgery. Laryngoscope. 1992; 102 (Suppl 57): 1-18.

5. Hopkins C, Slack R, Lund V, Brown P, Copley $L$, Browne J. Long-term outcomes from the English national comparative audit of surgery for nasal polyposis and chronic rhinosinusitis. Laryngoscope. 2009; 119: 24592465.

6. Rudnick EF, Mitchell RB. Improvements in quality of life in children after surgical therapy for sinonasal disease. Otolaryngol Head Neck Surg. 2006; 134: 737-740.
7. Siedek V, Stelter K, Betz CS, Berghaus A, Leunig A. Functional endoscopic sinus surgery--a retrospective analysis of 115 children and adolescents with chronic rhinosinusitis. Int J Pediatr Otorhinolaryngol. 2009; 73: 741-745.

8. Hebert RL, Bent JP, III. Meta-analysis of outcomes of pediatric functional endoscopic sinus surgery. Laryngoscope. 1998; 108: 796-799.

9. Fokkens WJ, Lund VJ, Mullol J, Bachert C, Alobid I, Baroody F, et al. EPOS 2012: European position paper on rhinosinusitis and nasal polyps 2012. A summary for otorhinolaryngologists. Rhinology. 2012; 50: 1-12.

10. Lusk RP, Bothwell MR, Piccirillo J. Long-term follow-up for children treated with surgical intervention for chronic rhinosinusitis. Laryngoscope. 2006; 116: 2099-2107.

11. Rudnick EF, Mitchell RB. Long-term improvements in quality-of-life after surgical therapy for pediatric sinonasal disease. Otolaryngol Head Neck Surg. 2007; 137: 873-877.

12. Manning SC. Surgical management of sinus disease in children. Ann Otol Rhinol Laryngol. Suppl 1992; 155: 42-45.

13. Ramadan HH. Relation of age to outcome after endoscopic sinus surgery in children. Arch Otolaryngol Head Neck Surg. 2003; 129: 175-177.

14. Lazar RH, Younis RT, Long TE. Functional endonasal sinus surgery in adults and children. Laryngoscope. 1993; 103: 1-5.

15. Gross CW, Gurucharri MJ, Lazar RH, Long TE. Functional endonasal sinus surgery (FESS) in the pediatric age group. Laryngoscope. 1989; 99: 272-275.

16. Tsukidate T, Haruna S, Fukami S, Nakajima I, Konno W, Moriyama H. Long-term evaluation after endoscopic sinus surgery for chronic pediatric sinusitis with polyps. Auris Nasus Larynx. 2012; 39: 583-587.

17. Ramsey B, Richardson MA. Impact of sinusitis in cystic fibrosis. J Allergy Clin Immunol. 1992; 90: 547-552.

18. Albritton FD, Kingdom TT. Endoscopic sinus surgery in patients with cystic fibrosis: an analysis of complications. Am J Rhinol. 2000; 14: 379-385.

19. Yung MW, Gould J, Upton GJ. Nasal polyposis in children with cystic fibrosis: a longterm follow-up study. Ann Otol Rhinol Laryngol. 2002; 111: 1081-1086.

20. Rosbe KW, Jones DT, Rahbar R, Lahiri T, Auerbach AD. Endoscopic sinus surgery in cystic fibrosis: do patients benefit from sur- 
gery? Int J Pediatr Otorhinolaryngol. 2001; 61: 113-119.

21. Becker SS, de AA, Bomeli SR, Han JK, Gross CW. Risk factors for recurrent sinus surgery in cystic fibrosis: review of a decade of experience. Am J Rhinol. 2007; 21: 478-482.

22. Cornet ME, Reinartz SM, Georgalas C, van SE, Fokkens WJ. The microdebrider, a step forward or an expensive gadget? Rhinology. 2012; 50: 191-198.

23. Piccirillo J. Psychometric and clinimetric validity of the 31 -item rhinosinusitis out- come measure (RSOM-31). Am J Rhinol. 1995; 9: 297-306.

24. Akinbami LJ, Moorman JE, Garbe PL, Sondik EJ. Status of childhood asthma in the United States, 1980-2007. Pediatrics. 2009; 123: Suppl 3: S131-S145.

25. Mallol J, Crane J, von ME, Odhiambo J, Keil U, Stewart A. The International Study of Asthma and Allergies in Childhood (ISAAC) Phase Three: A global synthesis. Allergol Immunopathol. 2013; 41: 73-85.
Marjolein E. Cornet

Department of Otorhinolaryngology

Academic Medical Centre Amster-

dam

1100 DD Amsterdam

The Netherlands

Tel: $+31-20-5664534$

E-mail:m.e.cornet@amc.uva.nl

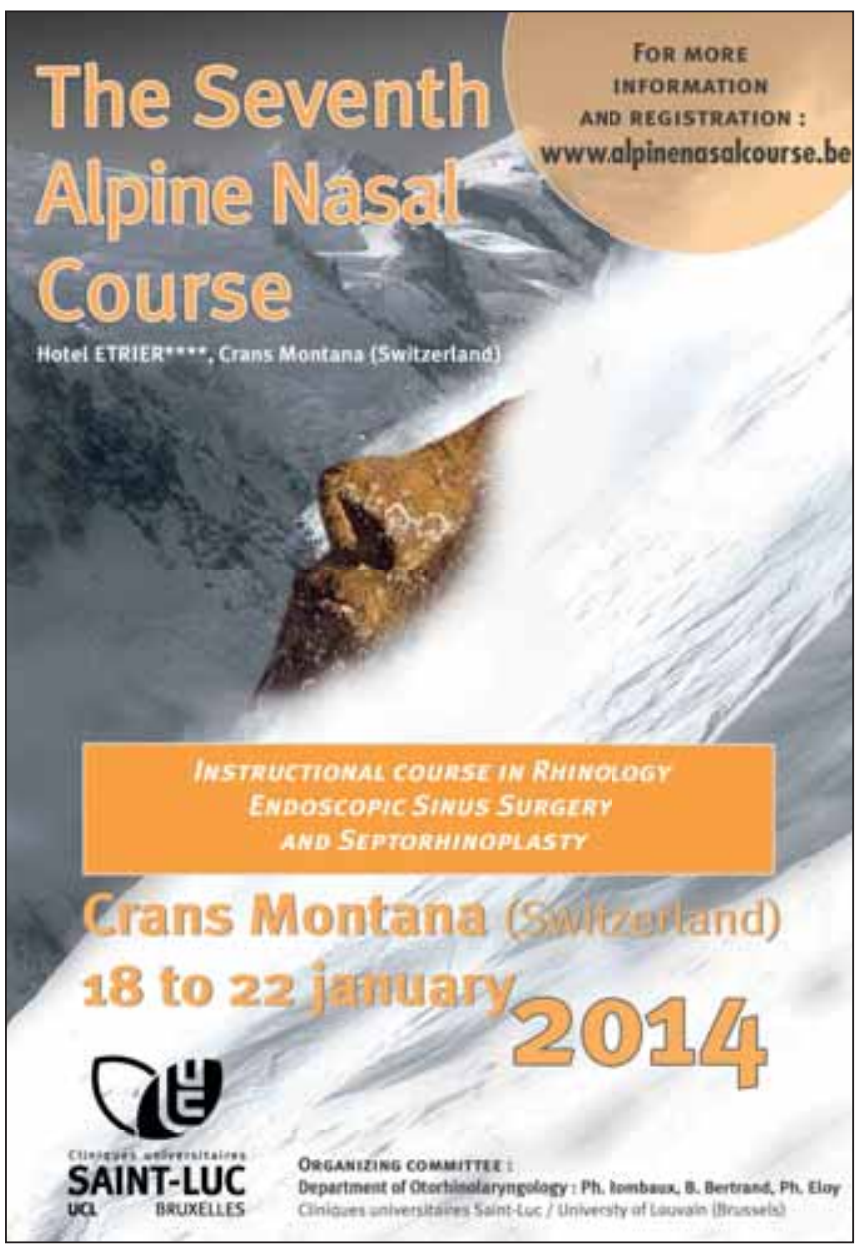

